\title{
A New Smoothing Algorithm and its Application in Gravitational Lensing ${ }^{\star}$
}

\author{
YANG Yi-bin ${ }^{1,2} \triangle$ LI Guo-liang ${ }^{3} \quad$ Alessandro B. Romeo ${ }^{4}$ \\ ${ }^{1}$ Center for Astrophysics, University of Science and Technology of China, Hefei 230026 \\ ${ }^{2}$ Purple Mountain Observatory, Chinese Academy of Sciences, Nanjing 210008 \\ ${ }^{3}$ Shanghai Astronomical Observatory, Chinese Academy of Sciences, Shanghai 200030 \\ ${ }^{4}$ Onsala Space Observatory, Chalmers University of Technology, SE-43992 Onsala, Sweden
}

\begin{abstract}
Based on the DWT (discrete wavelet transform) method, we propose a new smoothing algorithm for computing surface densities from 3D numerical simulation samples. To check its effectiveness, we have applied this algorithm to two Monte-Carlo samples of gravitational lens simulation with different mass resolutions, generated from the isothermal ellipsoid model of dark matter halos. The calculated results indicate that this algorithm can reconstruct accurately the surface density distribution of the gravitational lens simulation sample, and that the lens caustics and critical curves derived from the surface densities agree well with the theoretical curves. We have compared the results calculated by using 3 different wavelet bases (Daub4, Daub6 and B-spline 3th), and identified the best one. Without sacrificing its smoothing capability, this algorithm has a very fast computing speed, suitable for later N-body numerical simulations, which require even higher resolutions.
\end{abstract}

Key words: gravitational lensing - methods: numerical values

\section{INTRODUCTION}

In recent years, accompanying the progress in observational techniques, gravitational lensing has become an important tool for the studies of galaxies and cosmology. The superiority

Received 2007-11-05; revised version 2008-02-20

* A translation of Acta Astron. Sin. Vol. 49, No. 4, pp. 359-369, 2008

$\triangle$ ybyang@mail.ustc.edu.cn 
of gravitational lensing is that we can directly acquire from it information about the mass distribution in the universe. It has been widely used in various research fields of astronomy. It is an important tool to explore the distribution of dark matter in the universe ${ }^{[1,2]}$. It can be used to measure the mass distributions in galaxies and galaxy clusters $^{[3,4]}$, to acquire statistical information on large-scale cosmic structures for setting limits on the cosmological parameters ${ }^{[5,6]}$, and can be used as well for studying the substructure problem in the hierarchically clustering theory ${ }^{[7-10]}$.

The gravitational lens in the universe has a much more complex structure than in various theoretical mass model distributions, such as asymmetry and substructures. So, the N-body numerical simulation is the best way to study accurately the real properties of gravitational lens. When we study the gravitational lens by N-body numerical simulations, the simulated lens is constructed by discrete particles in a box, corresponding to a smooth mass distribution in real space. To study the properties of the lens generated by numerical simulation under the thin-lens approximation, we have to obtain first the projected surface density field on the lens plane from the simulated 3D density field, before the lens properties, such as the lens potential, deflection angle, image magnification and others, are derived. Hence, a smoothing algorithm which can construct accurately the lens surface densities is extremely important in the study of gravitational lensing.

There have been many different smoothing algorithms used for the study of gravitational lensing, including the commonly adopted CIC (Cloud-In-Cell) format ${ }^{[11]}$ and TSC (Triangular Shaped Clouds) format. Some researchers have used more complex smoothing algorithms, such as the Gaussian kernel function ${ }^{[12,13]}$ and SPH (Smoothed Particle Hydrodynamics) kernel function ${ }^{[14,15]}$. Some smoothing algorithms adopt a smoothing scale of fixed length, for example the CIC, TSC or Gaussian kernel function. When kernel functions of this kind are used, we can prove easily that identical results are obtained whether we use the so-called 2D smoothing (where we first project the particles onto the lens plane, then calculate the surface densities by smoothing), or the 3D smoothing (where we first calculate the $3 \mathrm{D}$ volume density field, then integrate along the direction vertical to the lens plane to obtain the surface densities). Therefore, for the fixed-scale smoothing algorithm, we need only to use the 2D smoothing, thus saving much computing time. However, in some smoothing algorithms such as the SPH kernel function, the smoothing scale is not fixed, rather, it is self-adaptive: a short smoothing scale is adopted in high-density regions, a long one, in low-density regions. Compared to the $2 \mathrm{D}$ smoothing, the $3 \mathrm{D}$ smoothing can eliminate some of the projection noises, but it calls for a much greater computer time.

Since the 1990s, the discrete wavelet transform (DWT) has been applied to the statistical studies in cosmology ${ }^{[16-19]}$. The main advantage of the DWT is that it can provide us with the statistical properties in both the position space and wave-number space. Based on the DWT method, we will propose a new smoothing algorithm in this paper, and apply it to calculate the surface densities of gravitational lens.

The structure of this paper is as follows. In the second part, we will introduce the smoothing algorithm and the noise-elimination program that we use; then, in the third part, the algorithm is verified by using the Monte-Carlo numerical simulation sample; and finally, conclusions are given in the fourth part. 


\section{THE ALGORITHM}

We will discuss the algorithm by separating it into two parts. First, we describe briefly the wavelet algorithm for smoothing a 3D sample, then we introduce the algorithm for noise elimination.

\subsection{Wavelet Smoothing Algorithm}

For simplicity, we discuss the $1 \mathrm{D}$ case. For a given density field $\rho(x)(0<x<L)$, we can decompose it to ${ }^{[20]}$

$$
\rho(x)=\sum_{\mathrm{l}=0}^{2^{J}-1} \epsilon_{J, l} \phi_{J}, l(x)+\sum_{\mathrm{j}=\mathrm{J}}^{\infty} \sum_{\mathrm{l}=0}^{2^{j}-1} \tilde{\epsilon}_{\mathrm{j}, \mathrm{l}} \psi_{j}, l(x),
$$

in which $\epsilon_{J, l}$ represents the scale function coefficients (SFCs, in brief), $\phi_{J, l}(x)$ is the scale function, $\tilde{\epsilon}_{j, l}$ represents the wavelet function coefficients (WFCs, in brief), $\psi_{j, l}$ is the wavelet function. $J$ and $j$ are positive integers representing the scales, corresponding to the scales $L / 2^{J}$ and $L / 2^{j}$. $l$ is the integer indicating the position, corresponding to the spatial range $l L / 2^{j}<x<(l+1) L / 2^{j}$. The scale function can be given by its parent function $\phi(x)$ :

$$
\phi_{\mathrm{J}, 1}(x)=\left(\frac{2^{J}}{L}\right)^{1 / 2} \phi\left(2^{J} x / L-l\right),
$$

and $\epsilon_{J, l}$ can be calculated from the next formula:

$$
\epsilon_{\mathrm{J}, \mathrm{l}}=\int_{0}^{L} \rho(x) \phi_{\mathrm{J}, 1}(x) d x
$$

For the given particle distribution, we can express it in the following form:

$$
\rho(x)=\sum_{\mathrm{i}=1}^{N_{\mathrm{g}}} w_{\mathrm{i}} \delta^{D}\left(x-x_{\mathrm{i}}\right),
$$

in which $N_{\mathrm{g}}$ is the total number of particles, $\left\{x_{\mathrm{i}}\right\}$ is the position of the $i$-th particle $(0<$ $\left.x_{i}<L\right), \omega_{i}$ is the weight of the $i$-th particle, $\delta^{D}$ is the Dirac- $\delta$ function. The particles can be either actually observed galaxies, or particles in the numerical simulation sample. Substituting Eq.(4) into Eq.(1) and Eq.(3), we obtain:

$$
\rho(x)=\rho^{J} x+\sum_{\mathrm{j}=\mathrm{J}}^{\infty} \sum_{\mathrm{l}=0}^{2^{\mathrm{j}}-1} \tilde{\epsilon}_{\mathrm{j}, 1}^{\mathrm{g}} \psi_{\mathrm{j}, 1}(x),
$$

in which $\rho^{J}$ is:

$$
\rho^{J}(x)=\sum_{\mathrm{l}=0}^{2^{\mathrm{J}}-1} \epsilon_{\mathrm{J}, \mathrm{l}} \phi_{\mathrm{J}, \mathrm{l}}(x),
$$

the scale function coefficient $\epsilon_{J, l}$ is:

$$
\epsilon_{\mathrm{J}, \mathrm{l}}=\sum_{i=1}^{N_{\mathrm{g}}} w_{\mathrm{i}} \phi_{\mathrm{J}, \mathrm{l}}\left(x_{\mathrm{i}}\right)
$$


and $\rho^{\mathrm{J}}$ is the smoothing of $\rho(x)$ on scale $J$. When $J$ is sufficiently large, $\rho^{J}(x)$ will be a very good approximation of $\rho(x)$. We also have

$$
\sum_{\mathrm{l}=0}^{2^{\mathrm{j}}-1} \frac{L}{2^{\mathrm{j}}} \epsilon_{\mathrm{j}, 1}^{g}=\sum_{\mathrm{i}=1}^{N_{\mathrm{g}}} w_{\mathrm{i}} .
$$

We can find that the mass allocated to the $l$-th grid point by the $i$-th particle is: $\left(L / 2^{j}\right) \omega_{i}$ $\phi_{J, l}\left(x_{i}\right)$. The scale function coefficients SFCs are actually a kind of mass re-allocation of the density field $\rho(x)$.

The procedure to calculate the surface densities of the gravitational lens using this algorithm is as follows: First, we have all the particles of the lens projected vertically to the given lens plane to obtain a 2D distribution, then we calculate the scale function coefficients SFCs by Eq.(7). After the SFCs are obtained, we calculate the surface density at an arbitrary point on the lens plane by Eq.(5).

\subsection{Wavelet De-noising Algorithm}

We know that compared to the 3D smoothing, the 2D smoothing will introduce some extra projection noise. We can eliminate this noise using the wavelet method. The wavelet method is, in fact, an effective tool for noise elimination, and has widely been applied to the digital signal and image processing and other fields. In our derivation of the surface densities of the gravitational lens, we adopt the wavelet method developed by Romeo et al. ${ }^{[21,22]}$ to eliminate noise.

The process of noise elimination can be described as follows. If the data (for example the data of numerical simulations) to be processed contain Poisson noise, then a preprocessing should be done first. Using the Anscombe transform ${ }^{[23]}$, the data $Y_{P}$ with Poisson noise can be converted to the data $Y_{G}$ which contain only Gaussian white noise of variance $\sigma_{G}=1$ :

$$
Y_{\mathrm{G}}=2 \sqrt{Y_{\mathrm{P}}+\frac{3}{8}}
$$

Then, we make the fast wavelet transform on the preprocessed data. The fast wavelet transform has a very important feature: After the wavelet transform is performed on a noisy data, the major part of the information carried by the data will be contained in a few large wavelet coefficients, while the noise will be contained in many tiny ones. If we set a suitable threshold value, and have all the wavelet coefficients less than this threshold set to zero, then after the inverse transform, we can obtain a set of new data that is basically free of the original noise.

Hence, the key step in the whole process of noise elimination is to select a suitable threshold value of the wavelet coefficients. We shall deal with it separately for several different cases.

The threshold value $T$ is proportional to the standard deviation of the noise, $\sigma$, with factor of proportionality, $K$, determined by the size $N_{\mathrm{d}}$ of the data:

$$
T=K\left(N_{\mathrm{d}}\right) \sigma \text {. }
$$

If the data have not been preprocessed, and the standard deviation is unknown (noise is mainly Gaussian white noise), then the standard deviation $\sigma$ can be estimated from the 
median absolute deviation (MAD) of a part of the wavelet coefficients $D_{\mathrm{i}}\left(N_{\mathrm{d}} / 2\right)$ :

$$
\sigma \simeq \frac{1}{0.6745} \operatorname{MAD}\left[D_{\mathrm{i}}\left(N_{\mathrm{d}} / 2\right)\right]
$$

If the noise is dominated by Poisson noise, then after the preprocessing, its standard variance can be estimated as:

$$
\sigma \simeq 1
$$

For $K\left(N_{d}\right)$, there are two different estimates. The first is:

$$
K\left(N_{\mathrm{d}}\right)=\sqrt{2 \ln N_{\mathrm{d}}} .
$$

This estimate has a rather high efficiency of noise elimination, but probably loses some original information of the data, so it is not very safe. The other one is:

$$
K\left(N_{\mathrm{d}}\right) \simeq\left\{\begin{array}{ll}
0 & \text { if } N_{\mathrm{d}} \leq 32 \\
0.3936+0.1829 \log _{2} N_{\mathrm{d}} & \text { if } N_{\mathrm{d}}>32
\end{array} .\right.
$$

For processing the numerical simulation data, we generally select the latter. After obtaining the threshold value, we can treat the wavelet coefficients by two different means. One is the so-called hard processing:

$$
\bar{W}_{\mathrm{i}}=\left\{\begin{array}{ll}
0 & \text { if }\left|W_{\mathrm{i}}\right| \leq T \\
W_{\mathrm{i}} & \text { if }\left|W_{\mathrm{i}}\right|>T
\end{array},\right.
$$

in which $W_{i}$ represents the wavelet coefficient, $\bar{W}_{i}$, the same after the processing. In the hard processing, those wavelet coefficients that are less than the threshold value are taken to be zero, and the other coefficients are kept unchanged. The corresponding soft processing is:

$$
\bar{W}_{\mathrm{i}}=\left\{\begin{array}{ll}
0 & \text { if }\left|W_{\mathrm{i}}\right| \leq T \\
\operatorname{sign}\left(\mathrm{W}_{\mathrm{i}}\right)\left(\left|\mathrm{W}_{\mathrm{i}}\right|-\mathrm{T}\right) & \text { if }\left|W_{\mathrm{i}}\right|>T
\end{array} .\right.
$$

In the soft processing, all the wavelet coefficients greater than the threshold value $T$ will have $T$ subtracted. For numerical simulation data, the soft processing will bring certain biases to the de-noised data, so the hard processing is usually selected.

After processing the wavelet coefficients, we make an inverse fast wavelet transform to obtain the de-noised data. Of course, if the original data have been preprocessed, then the data should be restored correspondingly by the inverse Anscombe transform. It should be noted that the Anscombe transform will bring a bias to the data, but this bias can be estimated analytically as:

$$
\text { bias } \simeq-\frac{1}{4}\left(1-\frac{1}{N_{\mathrm{d}}}\right) \sigma^{2} .
$$

Thus, we need only to subtract this bias from all the de-noised data.

Performing the DWT decomposition on the particles of the lens simulation sample, we obtain a matrix of SFCs. By using the de-noising algorithm in this section, we can make the noise elimination on this matrix of SFCs. As the matrix of SFCs contains the information of the density field, so the noise elimination of SFCs is equivalent to the noise elimination of the whole density field. Then, using the de-noised matrix of SFCs, the density field can be reconstructed. 


\section{NUMERICAL TEST}

The isothermal ellipsoid model proposed by Kormann ${ }^{[24]}$ and Keeton ${ }^{[25]}$ is the most commonly used model of gravitational lens. It has an analytical critical curve and caustics. We will employ it to test the smoothing algorithm.

The density distribution of an isothermal ellipsoid is:

$$
\rho=\frac{v_{\mathrm{c}}{ }^{2}}{4 \pi G q_{3}} \frac{e}{\sin ^{-1} e} \frac{1}{s^{2}+x^{2}+y^{2}+z^{2} / q_{3}^{2}},
$$

in which $v_{\mathrm{c}}$ is the halo's rotational velocity, $G$ is the gravitational constant, $s$ is the radius of the ellipsoid's central core, the $\mathrm{X}$-axis and $\mathrm{Y}$-axis of the ellipsoid are equally long, $q_{3}$ is the ratio between the short Z-axis and the two equally long axes, and the projection ellipticity is $e=\sqrt{1-q_{3}^{2}}$. We take $v_{c}=1500 \mathrm{~km} \mathrm{~s}^{-1}, s=20 \mathrm{kpc}$ and $q_{3}=0.5$.

With the Monte-Carlo method, we can produce simulation samples of the density distribution satisfying Eq.(18). The size of the cubic box at the lens position is taken to be $4 \mathrm{Mpc}$. The redshifts of the lens and source are respectively taken to be 0.3 and 1.0 , and the projection direction is along the $\mathrm{Y}$-axis. Two groups of simulation samples with different mass resolutions were produced: a sample with a high mass resolution containing $N_{p}=2 \times 10^{6}$ particles, each with mass $M_{p}=4.375 \times 10^{8} h^{-1} M_{\odot}$; and a sample with a low mass resolution containing $N_{p}=2 \times 10^{5}$ particles, each with $M_{p}=4.375 \times 10^{9} h^{-1} M_{\odot}$. The unit of the Hubble constant is taken to be $100 \mathrm{~km} \mathrm{~s}^{-1} \mathrm{Mpc}^{-1}$.

With the above algorithm we calculated the surface density $\kappa$ of the simulation samples, on a $1024 \times 1024$ grid. For each sample, we made calculations with 3 different wavelet bases, Daub4, Daub6 and B-spline 3th. Then we calculated the lens caustics and the critical curves from the obtained surface density fields.

The procedure to calculate the critical curve and caustics is as follows: First, using the fast Fourier transform, we calculated the deflection potential at every grid point of the density field. Then, we calculated the magnification at every grid point to obtain a mapping from the image plane to the source plane. Finally, judging by the criterion that the sign of magnification should change on the two sides of the critical curve, the critical curve and caustics were determined ${ }^{[26]}$.

Fig.1 shows the lens' surface density distribution calculated for the sample with a low mass resolution. The plotted points in this figure represent the mean surface densities in the intervals between two adjacent ellipse radii, the solid line indicates the theoretical values calculated according to Eq.(18). The relative errors are given at the bottom of each panel. We see that all the 3 wavelet bases can fairly fit the theoretical curve. But in the area close to the center, the Daub4 wavelet base does not reproduce the surface densities very well, and the relative errors are rather large. On the other hand, the B-spline 3th and Daub6 wavelet bases can reconstruct this part of surface densities quite well. On the whole, the result of the B-spline 3th wavelet base is not so smooth as those of the Daub4 and Daub6. So generally speaking, the Daub6 wavelet base is the best choice. It is noteworthy that these results are obtained by using the wavelet de-noising algorithm. In order to show the effect of noise, we also calculated a set of surface densities, in which the noise was not eliminated in the calculation. 

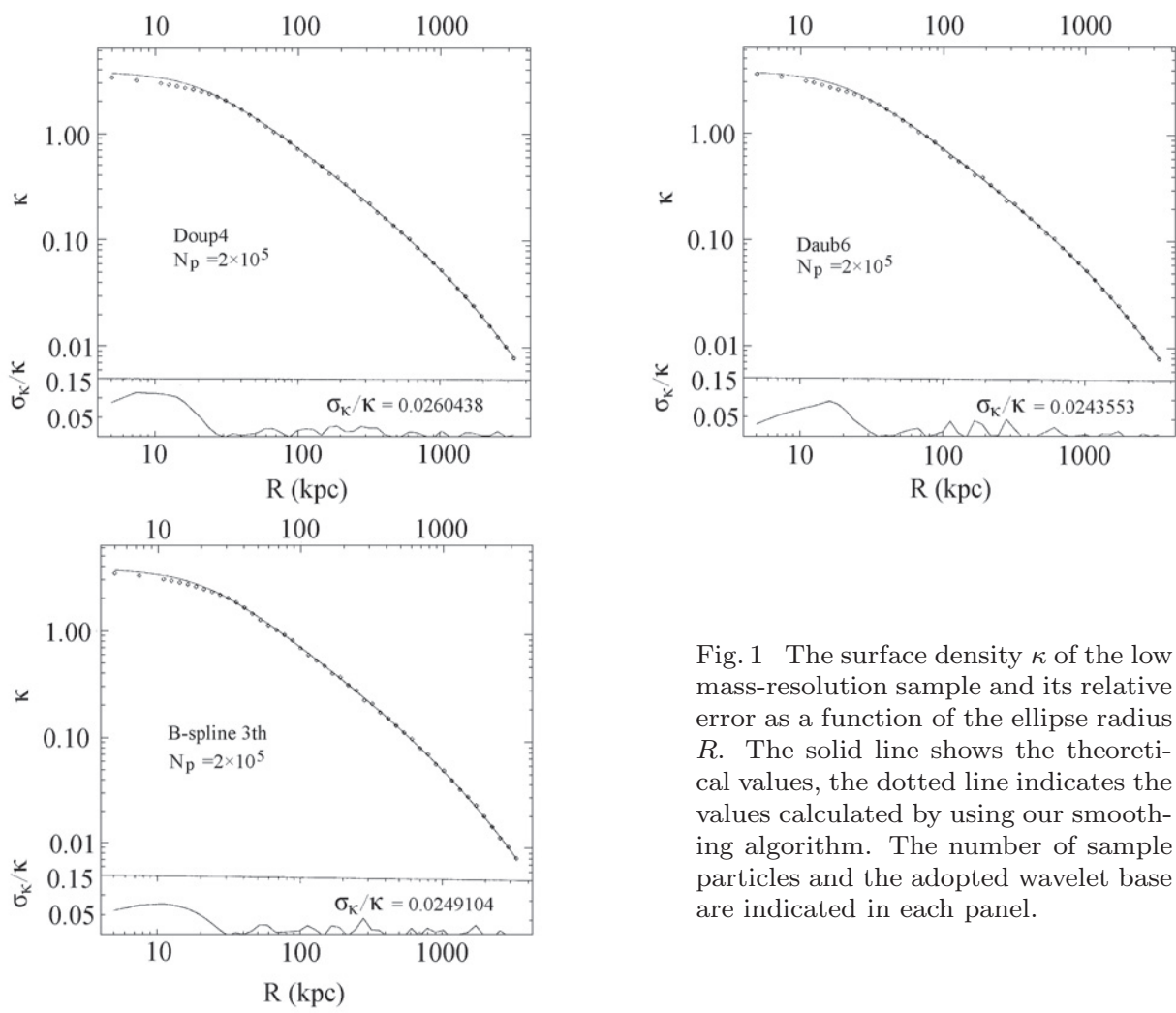

Fig. 1 The surface density $\kappa$ of the low mass-resolution sample and its relative error as a function of the ellipse radius $R$. The solid line shows the theoretical values, the dotted line indicates the values calculated by using our smoothing algorithm. The number of sample particles and the adopted wavelet base are indicated in each panel.

Fig. 2 is the same as the first panel of Fig. 1, based on using the Daub4 wavelet base, but without applying the wavelet algorithm of noise elimination. We note that after the de-noising, the noise level was greatly reduced, with no loss of information on the surface density field. It indicates that our wavelet de-noising algorithm can eliminate effectively the noise in the sample.

Fig. 3 displays the critical curves and caustics derived from the surface densities of the low mass-resolution sample. Because the Daub4 wavelet base can not fairly reconstruct the surface densities near the center, the calculated critical curve and caustics have rather large deviations from the theoretical curves, especially for the inner part of the critical curve. However, the Daub6 and B-spline 3th wavelet bases can fit rather well the theoretical curve, especially for the inner part of the critical curve, and the errors are far less than in the case of the Daub4 wavelet.

For the high mass-resolution Monte-Carlo sample, we have likewise calculated the projected surface densities and the corresponding critical curves and caustics. See Fig. 4 and Fig. 5. We see that the relative errors of the projected surface densities are far less here than in the low mass-resolution sample: the relative errors calculated by the 3 wavelet bases are all controlled to be below $2 \%$. The corresponding critical curves and caustics are very good and match the theoretical curves very well. Compared to the other two wavelet bases, the Daub6 wavelet base seems to give slightly more smooth results, almost coincident with the 
theoretical curve.

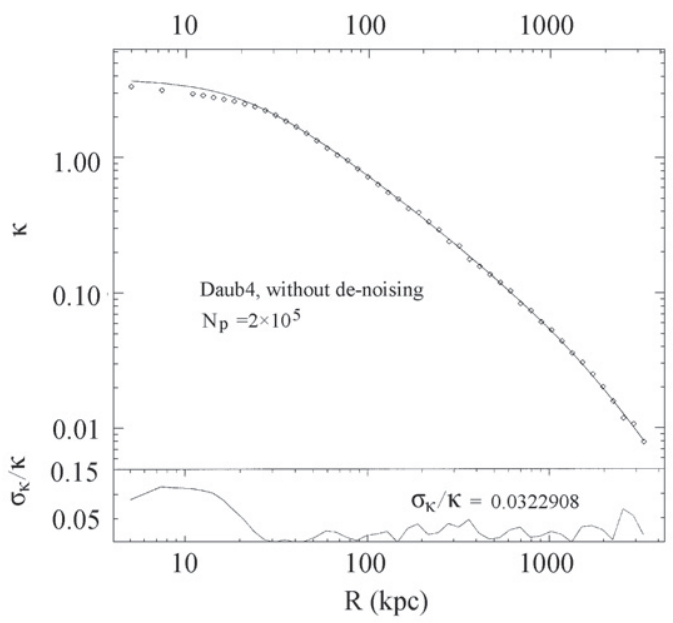

Fig. 2 Same as the first panel of Fig.1, but the wavelet de-noising algorithm has not been applied.
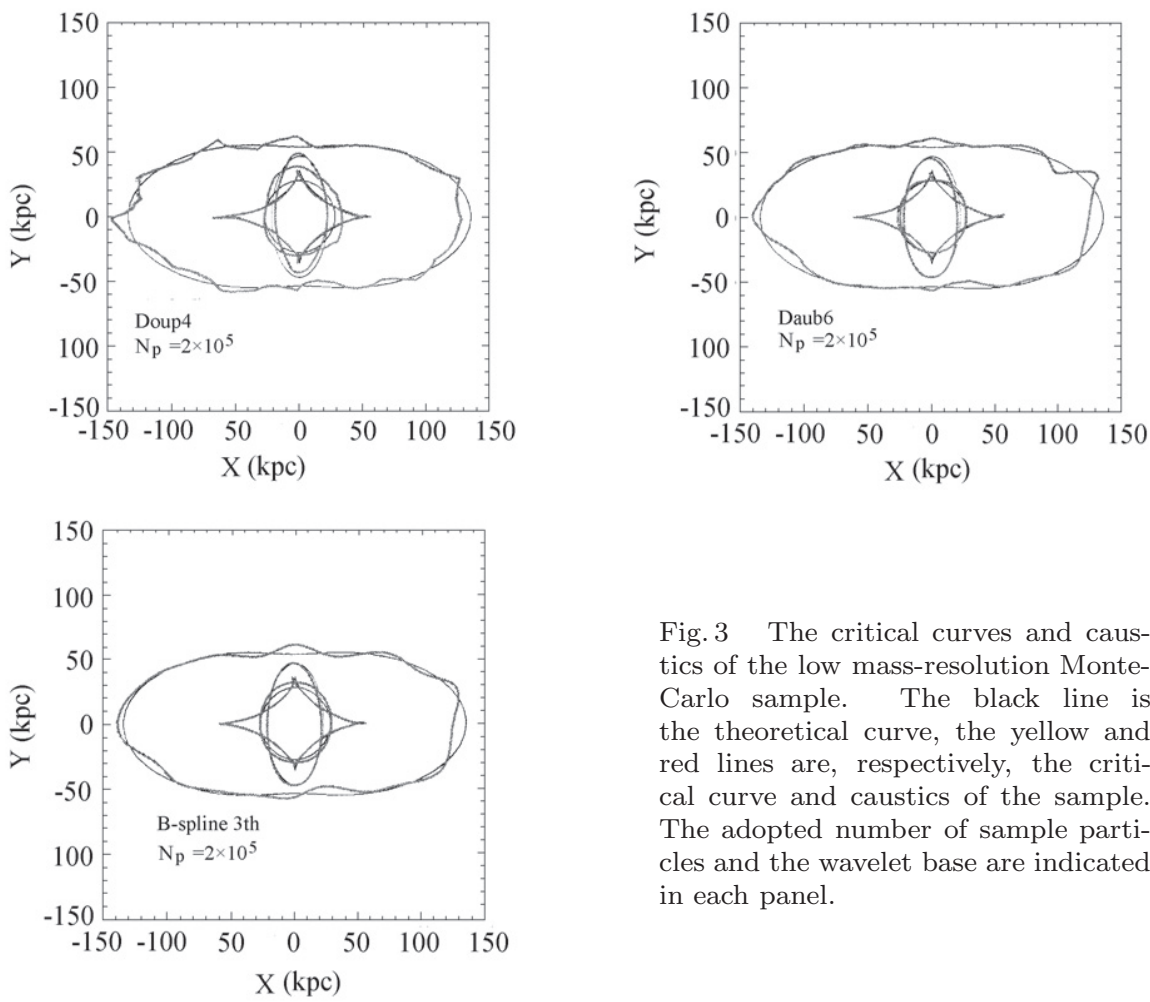

Fig. 3 The critical curves and caustics of the low mass-resolution MonteCarlo sample. The black line is the theoretical curve, the yellow and red lines are, respectively, the critical curve and caustics of the sample. The adopted number of sample particles and the wavelet base are indicated in each panel. 

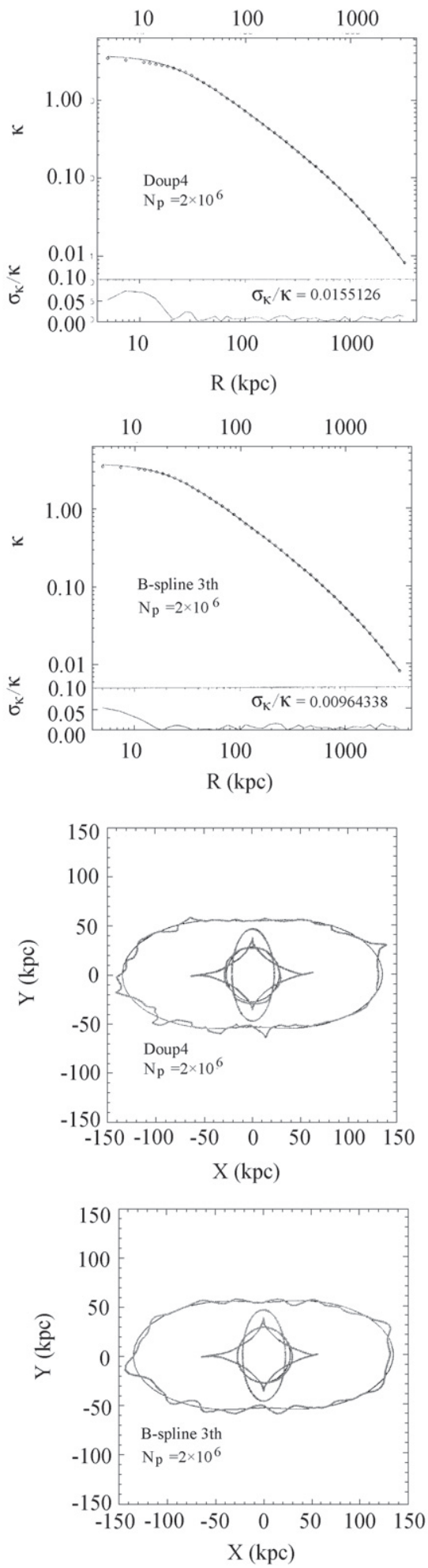

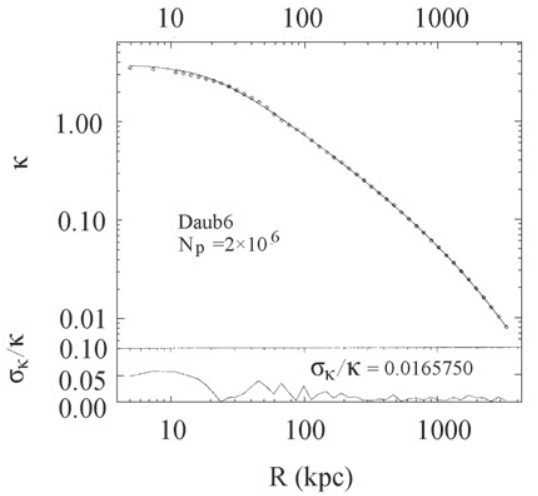

Fig. 4 The surface density $\kappa$ of the high mass-resolution sample and its relative error as a function of the ellipse vector $R$. The solid line represents the theoretical values, the dotted line, the results calculated using the smoothing algorithm. The adopted number of sample particles and the wavelet base are indicated in each panel.

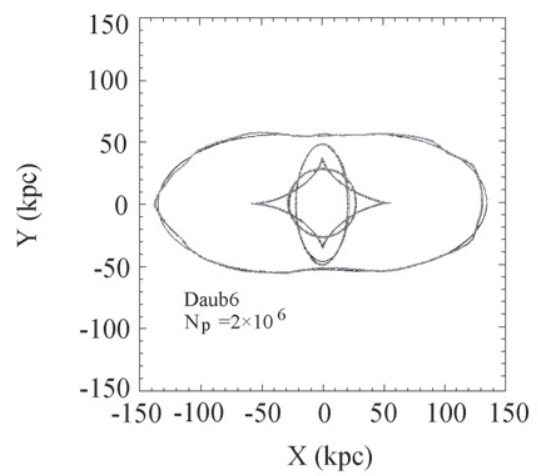

Fig. 5 The critical curves and caustics of the high mass-resolution MonteCarlo sample. The black line is the theoretical curve, the yellow and red lines are, respectively, the critical curve and caustics of the sample. The adopted number of sample particles and the wavelet base are indicated in each panel. 
For comparisons, we quote here the Monte-Carlo sample results obtained by Li G. L. et al. ${ }^{[15]}$ using the SPH3D smoothing algorithm. They used the same parameters as ours for the lens simulation, and only a slightly different number of particles from our high massresolution sample (namely, 1748982), so an approximate comparison can be made. Fig. 6 displays the lens' surface density distribution they calculated and Fig. 7, the corresponding critical curve. Compared with the result of our high mass-resolution sample, the errors of their surface densities are significantly larger, and the critical curve derived from the surface densities is also not so smooth as ours. It means that on the order of magnitude of $10^{6}$, the algorithm of ours is superior.

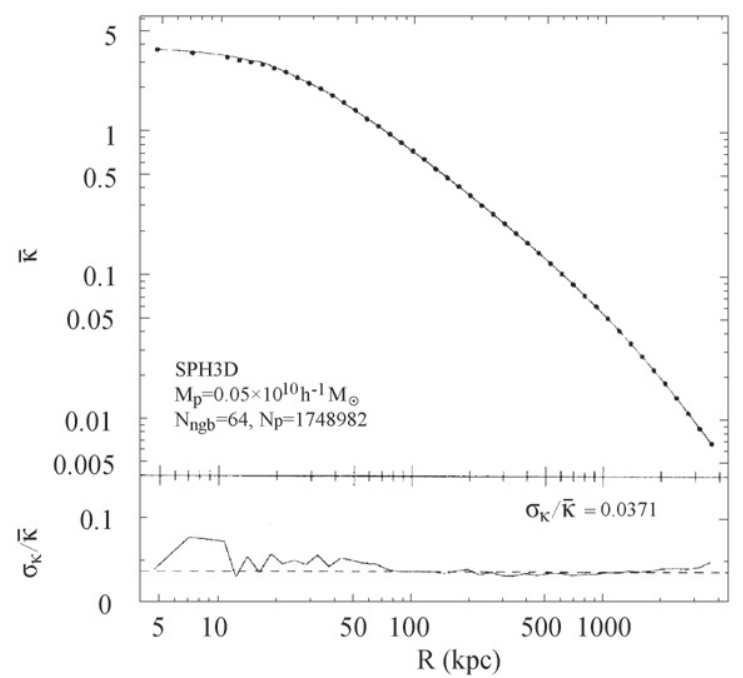

Fig. 6 The surface density $\kappa$ calculated by the SPH3D method and its relative error as functions of the ellipse radius $R^{[15]}$. The solid line shows the theoretical values, the dotted line shows the results calculated by the SPH smoothing algorithm. The adopted number of sample particles is indicated in the figure.

Generally, for the calculations of the lens simulation sample with a rather large number of sample particles (of the order of $10^{5}$ or $10^{6}$ ), our smoothing algorithm can fairly reconstruct the lens' surface density field. But if the number of sample particles is relatively small (below the order of $10^{5}$ ), then the calculated surface density field will have certain errors. With the improving computing power of the current computer hardware, the particle number adopted for modern numerical simulation studies can be made increasingly larger. For the N-body numerical simulations of gravitational lensing, the number of sample particles is commonly of the order of $10^{5}-10^{6}$ or even higher, very suitable for the application of our algorithm.

It is noteworthy that this algorithm has a very fast speed for the calculation of surface densities. We made our calculations with an Intel Itanium CPU, and our computing time was generally less than 20 seconds. By comparison, the SPH3D algorithm adopted by Li G. L. et al. needed a time as long as several minutes. Hence, for further simulations with particle numbers on the order of $10^{8}$ even $10^{9}$, our algorithm is superior. 


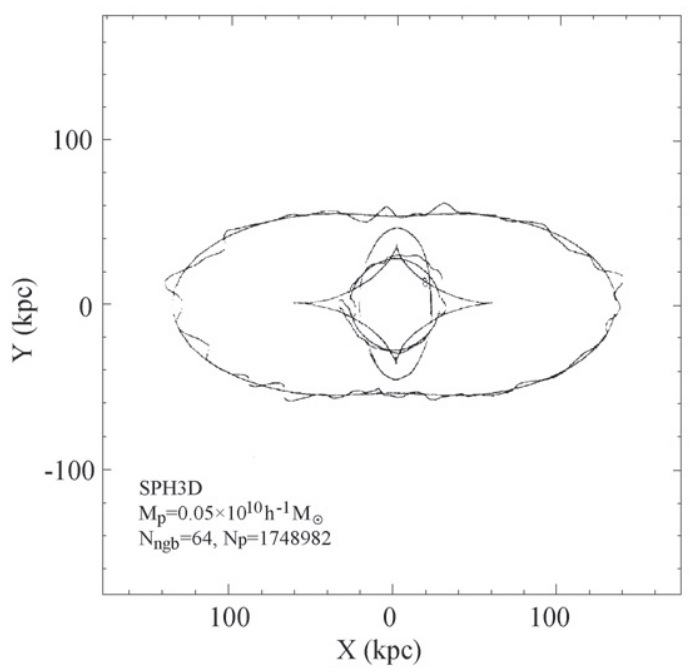

Fig. 7 The critical curve and caustics of the surface densities calculated by the SPH3D $\operatorname{method}^{[15]}$. The blue and red curves are the theoretical critical curve and caustics, the black curve is the critical curve of the sample.

\section{CONCLUSIONS}

We have introduced a smoothing algorithm, in which the DWT multi-scale analysis is used, and applied it to the study of gravitational lensing. For testing this algorithm, we have produced numerical simulation samples of an isothermal ellipsoid using the Monte-Carlo method. Two simulation samples with different mass resolutions were prepared. The calculated results indicate that this algorithm can reconstruct very well the surface density field of the high mass-resolution lens sample, and that the relative errors of the computed surface density field are controlled to a very low level. For the low mass-resolution sample, the algorithm can fairly reconstruct the surface density field as well. We compared three wavelet bases, the Daub4, Daub6 and B-spline 3th wavelet bases. The calculated results indicate that the Daub6 wavelet base is the best, its calculated surface density has the smallest relative error, and the critical curve and caustics then derived are also the best. We have compared our results with those calculated by Li G. L. et al. with the SPH3D method. For a sample with a size comparable to that of our high mass-resolution sample, their calculated surface densities have higher relative errors than ours, and their derived critical curve is also not as smooth as ours. This indicates that when the particle number is on the order of $10^{6}$, our algorithm is superior.

We will apply this algorithm to study the properties of the lens samples produced by N-body simulations. This work is in preparation.

\section{References}

1 Massey R., Rhodes J., et al., Nature, 2007, 445, 286

2 Massey R., Rhodes J., et al., ApJS, 2007, 172, 239

3 Gray E., Taylor N., et al., ApJ, 2006, 568, 141 
4 Gavazzi R., Mellier Y., et al., A\&A, 2004, 422, 407

5 Contaldi R., Hoekstra H., Lweis A., Phys. Rev. Lett., 2003, 90, 1308

6 Pedersen K., Dahle H., ApJ, 2007, 667, 26

7 Chiba M., ApJ, 2002, 565, 17

8 Dalal N., Kochanek C., ApJ, 2002, 572, 25

9 Metcalf R., Madau P., ApJ, 2001, 563, 9

10 Bradac M., Schneider P., et al., A\&A, 2004, 423, 797

11 Amara A., et al., MNRAS, 2006, 367, 1367

12 Bartelmann M., Huss A., Colberg J. M., et al., A\&A, 1998, 330, 1

13 Bradac M., et al., A\&A, 1998, 388, 373

14 Li G. L., Mao S., Jing Y. P., et al., ApJ, 2005, 635, 795

15 Li G. L., Mao S., Jing Y. P., et al., ApJ, 2006, 652, 43

16 Feng L. L., Deng Z. G., Fang L. Z., ApJ, 2000, 530, 53

17 Feng L. L., Fang L. Z., ApJ, 2000, 535, 519

18 Feng L. L., Pando J., Fang L. Z., ApJ, 2001, 555, 74

19 Feng L. L., ApJ, 2007, 658, 25

20 Fang L. Z., Thews R., Wavelet in Physics, Singapore: World Scientific, 1998

21 Romeo A. B., Horellou C., Bergh J., MNRAS, 2003, 342, 337

22 Romeo A. B., Horellou C., Bergh J., MNRAS, 2004, 354, 1208

23 Anscomber F., Biometrika, 1948, 35, 246

24 Kormann R., et al., A\&A, 1994, 284, 285

25 Keeton C., Kochanek C., ApJ, 1998, 495, 157

26 Bartelmann M., astro-ph/0304162 